\title{
Imaging Appearance of the Lateral Rectus-Superior Rectus Band in 100 Consecutive Patients without Strabismus
}

\author{
S.H. Patel, M.E. Cunnane, A.F. Juliano, M.G. Vangel, M.A. Kazlas, and G. Moonis
}

\begin{abstract}
BACKGROUND AND PURPOSE: The lateral rectus-superior rectus band is an orbital connective tissue structure that has been implicated in a form of strabismus termed sagging eye syndrome. Our purpose was to define the normal MR imaging and CT appearance of this band in patients without strabismus.
\end{abstract}

MATERIALS AND METHODS: Orbital MR imaging and CT examinations in 100 consecutive patients without strabismus were evaluated. Readers graded the visibility of the lateral rectus-superior rectus band on coronal TIWI, coronal STIR, and coronal CT images. Readers determined whether the band demonstrated superotemporal bowing or any discontinuities and whether a distinct lateral levator aponeurosis was seen. Reader agreement was assessed by $\kappa$ coefficients. Association between imaging metrics and patient age/sex was calculated by using the Fisher exact test.

RESULTS: The lateral rectus-superior rectus band was visible in $95 \%$ of coronal TIWI, $68 \%$ of coronal STIR sequences, and $70 \%$ of coronal CT scans. Ninety-five percent of these bands were seen as a continuous, arc-like structure extending from the superior rectus/levator palpebrae muscle complex to the lateral rectus muscle; $24 \%$ demonstrated superotemporal bowing; and in $82 \%$ of orbits, a distinct lateral levator aponeurosis was visible. Increasing patient age was negatively associated with lateral rectus-superior rectus band visibility ( $P=.03$ ), positively associated with lateral rectus-superior rectus band superotemporal bowing $(P=.03)$, and positively associated with lateral levator aponeurosis visibility $(P=.01)$.

CONCLUSIONS: The lateral rectus-superior rectus band is visible in most patients without strabismus on coronal TTWI. The age effect with respect to its visibility and superotemporal bowing could represent age-related connective tissue degeneration.

ABBREVIATIONS: LR-SR band = lateral rectus-superior rectus band

$\mathbf{T}$ he presence of stereotypic connective tissue structures within the orbit has been recognized on histologic studies for decades. ${ }^{1,2}$ More recent investigations have refined the anatomy and revealed the role these structures play in normal eye motility. ${ }^{3-7}$ Critical among these structures are the rectus muscle pulleys. ${ }^{8,9}$ The pulleys are collagenous rings within the Tenon fascia that encircle the rectus muscles near the globe equator. They inflect

Received September 29, 2013; accepted after revision February 19, 2014.

From the Departments of Radiology (S.H.P., M.E.C., A.F.J., G.M.) and Ophthalmology (M.A.K.), Massachusetts Eye and Ear Infirmary, Boston, Massachusetts; Biostatistics Center (M.G.V.), Massachusetts General Hospital, Boston, Massachusetts; and Department of Ophthalmology (M.A.K.), Boston Children's Hospital, Boston, Massachusetts.

Paper previously presented at: Annual Meeting of the American Society of Neuroradiology, May 18-23, 2013; San Diego, California.

Please address correspondence to Sohil H. Patel, MD, Department of Radiology, Massachusetts Eye and Ear Infirmary, 243 Charles St, Boston, Massachusetts, 02114; e-mail: spatel57@partners.org

http://dx.doi.org/10.3174/ajnr.A3943 rectus muscle paths in a manner analogous to the trochlea with respect to the tendon of the superior oblique muscle.

The rectus muscle pulleys are themselves interconnected by suspensory connective tissue bands that extend between each adjacent rectus muscle pulley. These connective tissue bands variably comprise collagen, elastin, smooth muscle, and fat. ${ }^{4}$

One connective tissue band that has garnered recent clinical interest is the lateral rectus-superior rectus band (LR-SR band). The LR-SR band is an arc-like collagenous band that connects the lateral rectus muscle to the superior muscle complex at the level of their respective pulleys (schematically represented in Fig 1). Degeneration of this band has been implicated in the development of 2 related forms of strabismus: heavy eye syndrome and sagging eye syndrome. ${ }^{10,11}$ Heavy eye syndrome affects elderly patients with high axial myopia. Patients present with esotropia (inward eye deviation) and hypotropia (downward eye deviation) due, in part, to inferior displacement of the lateral rectus muscle and nasal displacement of the superior rectus muscle. The staphylo- 


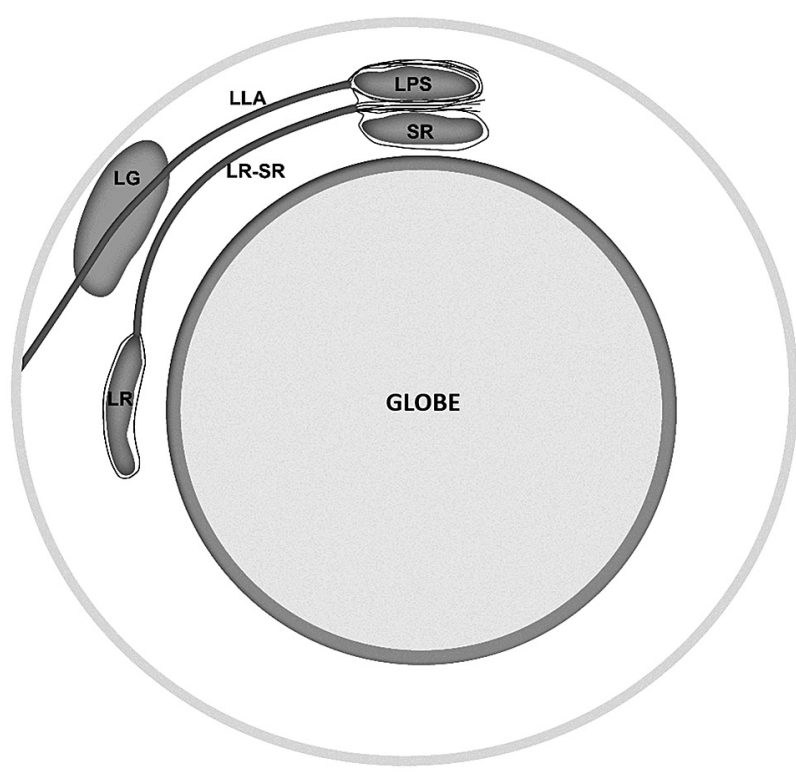

FIG 1. Schematic representation of orbital connective tissues within the superotemporal orbit. LG indicates lacrimal gland; LLA, lateral levator aponeurosis; LPS, levator palpebrae superioris; LR, lateral rectus muscle; SR, superior rectus muscle.

matous globes seen in these patients appear to produce this characteristic extraocular muscle displacement by mass effect. ${ }^{12}$ On the other hand, patients with sagging eye syndrome do not have high axial myopia (or staphylomas), yet they have a strabismus pattern similar to that of patients with heavy eye syndrome. In sagging eye syndrome, it is speculated that degeneration of the LR-SR band allows inferior displacement of the lateral rectus muscle and pulley. Evidence for this theory comes from 2 recent reports demonstrating LR-SR band degeneration in elderly patients with strabismus, using an experimental MR imaging technique in which a quasicoronal imaging plane was used and gaze direction was controlled. ${ }^{10,11}$

No prior studies have focused on defining the normal MR imaging appearance of the LR-SR band in a large cohort of patients without strabismus, to our knowledge. Moreover, no reports exist on the 3T MR imaging or CT appearance of the LR-SR band in clinical orbital imaging examinations. The purpose of this study was to define the normal appearance of the LR-SR band in patients without strabismus on clinical 3T MR imaging scans of the orbits. When available, the CT appearance of the band is also characterized.

\section{MATERIALS AND METHODS \\ Patient Selection}

Institutional review board approval for this Health Insurance Portability and Accountability Act-compliant retrospective review of patient data was obtained.

Patients who underwent orbital MR imaging examinations at our institution from June 2012 to October 2012 were candidates for inclusion in this study. The medical records and imaging examinations of these patients were reviewed. Patients were excluded from the study for any of the following: 1) strabismus, 2) high axial myopia (worse than -4.5 diopters), 3) staphyloma, 4) pathology affecting the location of the LR-SR band, and 5) lack of coronal nonfat suppressed T1 and/or coronal STIR images of the orbits. One hundred patients who underwent orbital MR imaging examinations from June 2012 to October 2012 met the study criteria and were included in this study. There were 60 women and 40 men. Patient ages ranged between 9 and 81 years (mean, $50 \pm$ 16 years). Patients underwent orbital imaging examinations for the following indications: mass/tumor $(n=27)$, unexplained vision loss or optic neuropathy $(n=25)$, suspected optic neuritis $(n=13)$, orbital infection/inflammation $(n=10)$, suspected ischemic optic neuropathy $(n=5)$, elevated intraocular pressure $(n=5)$, elevated intracranial pressure $(n=4)$, cranial nerve palsy $(n=3)$, and other $(n=8)$.

\section{Imaging Technique}

MR imaging examinations were performed on a $3 \mathrm{~T}$ Achieva (Philips Healthcare, Best, the Netherlands) MR imaging scanner using an 8-channel phased array coil. Coronal T1-weighted imaging of the orbits was performed with the following parameters: $\mathrm{TR} / \mathrm{TE}=659 / 11 \mathrm{~ms}, \mathrm{FOV}=165 \mathrm{~cm}^{2}$, section thickness $=3 \mathrm{~mm}$, gap $=0.3 \mathrm{~mm}$, matrix $=472 \times 469, \mathrm{NEX}=2$. Coronal STIR imaging of the orbits was performed with the following parameters: $\mathrm{TR} / \mathrm{TE}=3800 / 30 \mathrm{~ms}, \mathrm{FOV}=160 \mathrm{~cm}^{2}$, section thickness $=$ $3 \mathrm{~mm}$, gap $=0.6 \mathrm{~mm}$, matrix $=268 \times 259, \mathrm{NEX}=2$.

CT examinations were performed on a Somatom Sensation (Siemens, Erlangen, Germany) multidetector row CT scanner, at $120 \mathrm{kV}$ (peak) and $200 \mathrm{~mA}$, by using a pitch of $0.8 \mathrm{~mm}$, collimation of $0.6 \mathrm{~mm}$, reconstructed with $2-\mathrm{mm}$ coronal section thickness.

\section{Image Analysis}

The MR imaging examinations were evaluated by 2 independent reviewers: a fifth-year neuroradiology attending and a first-year neuroradiology fellow. The reviewers characterized the appearance of the LR-SR band on coronal non-fat-suppressed T1weighted images and coronal STIR sequences (the LR-SR band was not clearly visible in the axial and sagittal imaging planes). Each reader independently determined the following metrics (yes or no): 1) Is the LR-SR band visible on coronal non-fat-suppressed T1-weighted images? 2) Is the LR-SR band visible on coronal STIR images? 3) When visible, is the LR-SR band seen as a continuous, uninterrupted structure from the superior muscle complex to the lateral rectus? 4) When visible, does the LR-SR band demonstrate superotemporal bowing (indicated by extension of any component of the LR-SR band lateral to the lateral rectus muscle and/or superior to the superior muscle complex)? 5) Is a distinct lateral levator aponeurosis visible? If a CT scan through the orbits was available in any patient, LR-SR band visibility on CT was determined on the basis of coronal reformations. After independent collection of the data, the readers reviewed, in consensus, any case over which they disagreed on the T1WI visibility of the band and identified potential factors that led to discrepancies. To familiarize themselves with the appearance of the LR-SR band, the readers reviewed the available literature depicting the LR-SR band on imaging studies and consulted with an experienced strabismus surgeon (M.A.K.) to ensure correct identification of the band. 

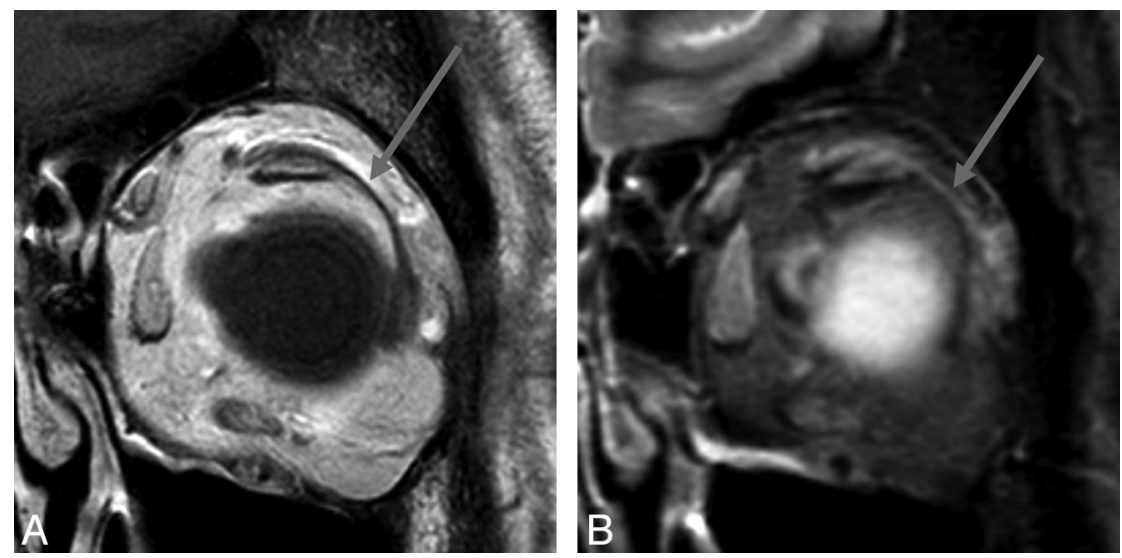

cant. Statistical analysis was performed with R software (http://www.r-project.org/). ${ }^{13}$

\section{RESULTS}

The LR-SR band was visible in $95 \%$ of orbits on coronal non-fat-suppressed T1weighted sequences and $68 \%$ of orbits on coronal STIR sequences (Fig 2). A CT scan through the orbits was available in 23 patients. The LR-SR band was visible in $70 \%$ of orbits on coronal CT reformations (Fig 3). For both readers, in every case in which the LR-SR band was visible on the STIR sequence or CT, it was also visible on the T1-weighted sequence.

FIG 2. MR imaging appearance of the LR-SR band in a 44-year-old woman. Coronal T1-weighted image $(A)$ and coronal STIR image $(B)$ of the left orbit demonstrate the LR-SR band (arrows) as a curvilinear structure extending from the superior margin of the lateral rectus muscle to the lateral margin of the superior rectus/levator palpebrae superioris muscle complex.

In $95 \%$ of cases, the LR-SR band was seen as an uninterrupted structure extending from the lateral margin of the su-

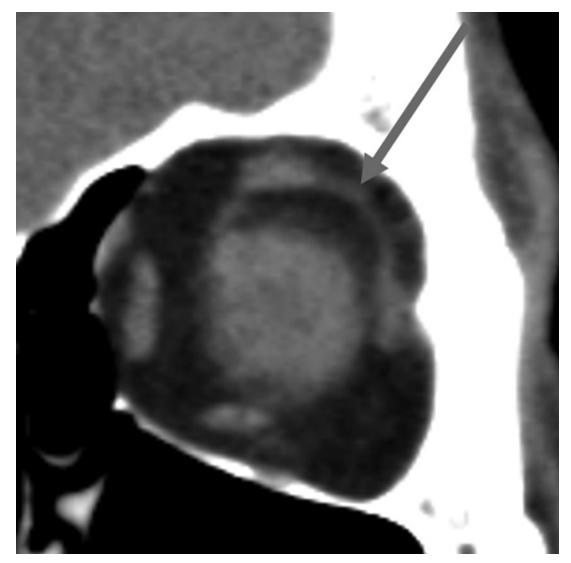

FIG 3. CT appearance of the LR-SR band in a 54-year-old woman. Coronal image of the left orbit demonstrates the LR-SR band (arrow).

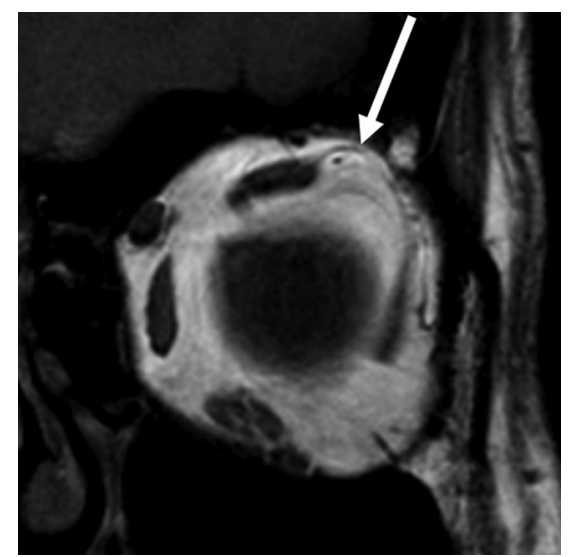

FIG 4. LR-SR band bowing in a 54-year-old woman. Coronal TIweighted image of the left orbit shows superior bowing of the LR-SR band (arrow), higher than the upper margin of the superior muscle complex.

\section{Statistical Analysis}

The imaging metrics determined by each reader were tabulated. Reader agreement was assessed by $\kappa$ coefficients. The Fisher exact test was used to determine correlation between age and sex with each of the imaging metrics. $P$ value $<.05$ was considered statistically signifiperior rectus/levator palpebrae muscle complex to the superior margin of the lateral rectus muscle (no discontinuities within the band). Twenty-four percent of visible LR-SR bands demonstrated superotemporal bowing (Fig 4). In most cases of superotemporal bowing, it was the posterior segment of the LR-SR band that was bowed. In $82 \%$ of orbits, a distinct lateral levator aponeurosis was visible (Fig 5).

Data per reader are displayed in Table 1 . The calculated $\kappa$ coefficients between readers were 0.38 for T 1 visibility, 0.26 for STIR visibility, 0.60 for CT visibility, 0.22 for continuous/discontinuous scores, 0.33 for superotemporal bowing scores, and 0.59 for lateral levator aponeurosis visibility scores. With respect to T1 visibility, of 200 cases, there were 12 disagreements between the readers; in all 12 cases, reader 1 detected the LR-SR band and reader 2 did not. With respect to STIR visibility, there were 74 disagreements between the readers; in 73 of these cases, reader 1 detected the LR-SR band and reader 2 did not. In 184 of 200 cases (92\%), the readers independently agreed that the LR-SR band was visible on T1WI. In 173 of the 200 cases (87\%), the readers independently agreed that the LR-SR band was both visible on T1WI and continuous.

After independent collection of the data, the readers reviewed, in consensus, the 12 cases over which they disagreed on T1WI visibility of the band. In consensus, the readers deemed the LR-SR band visible in 9 of the 12 cases and not visible in 3 of the 12 cases. The readers identified potential factors that led to discrepancies in these 12 cases. These included the following: close apposition of the LR-SR band to globe surface ( 5 cases); inhomogeneous orbital fat (4 cases); patient motion ( 2 cases); and a very thin band (1 case).

Data were further analyzed by using the Fisher exact test. A negative association was demonstrated between increasing quartiles of age and T1 visibility of the LR-SR band $(P=.03)$. A positive association was demonstrated between increasing quartiles of age and superotemporal bowing of the LR-SR band $(P=.03)$. A positive association was demonstrated between increasing quartiles of age and visibility of a distinct lateral levator aponeurosis $(P=.01)$. No significant correlation was demonstrated between age and the remaining imaging metrics. Table 2 displays imaging 

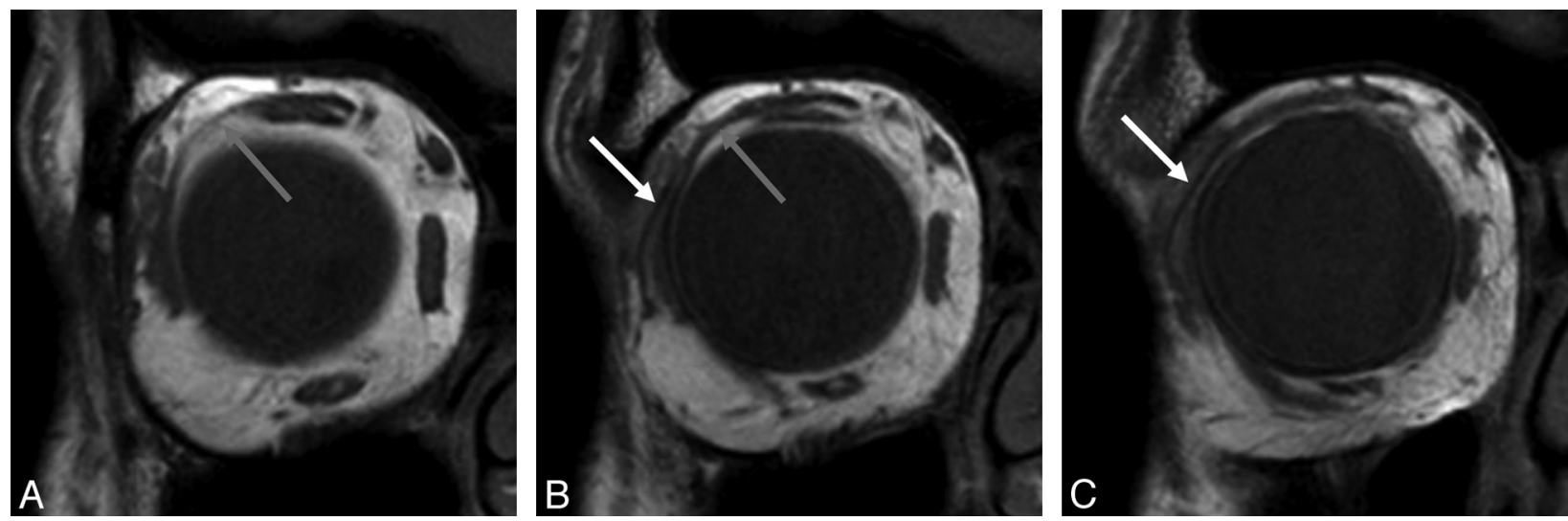

FIG 5. MR imaging appearance of the LR-SR band and the lateral levator aponeurosis in a 50-year-old man. Consecutive coronal TI-weighted images of the right orbit, from posterior $(A)$ to anterior $(C)$, show that the lateral levator aponeurosis (white arrows) lies superior and temporal to the LR-SR band (gray arrows) and traverses the lacrimal gland.

Table 1: Data per reader

\begin{tabular}{lcccccc}
\hline & $\begin{array}{c}\text { LR-SR Visible } \\
\text { on TIW1 }^{\text {a }}\end{array}$ & $\begin{array}{c}\text { LR-SR Visible } \\
\text { on STIR }^{\mathbf{a}}\end{array}$ & $\begin{array}{c}\text { LR-SR Visible } \\
\text { on CT }^{\mathbf{a}}\end{array}$ & $\begin{array}{c}\text { LR-SR } \\
\text { Continuous }^{\text {b }}\end{array}$ & $\begin{array}{c}\text { LR-SR } \\
\text { Bowing }^{\mathbf{c}}\end{array}$ & $\begin{array}{c}\text { LLA } \\
\text { Visible }^{\mathbf{d}}\end{array}$ \\
\hline Reader 1 & $98 \%$ & $86 \%$ & $63 \%$ & $97 \%$ & $22 \%$ & $79 \%$ \\
Reader 2 & $92 \%$ & $50 \%$ & $76 \%$ & $93 \%$ & $26 \%$ & $85 \%$ \\
\hline
\end{tabular}

Note:- LLA indicates lateral levator aponeurosis.

${ }^{\text {a }}$ The percentage of cases in which the LR-SR band was visible on the given imaging modality/sequence.

${ }^{b}$ When visible, the percentage of cases in which the LR-SR band formed a continuous structure extending from the superior muscle complex to the lateral rectus muscle (ie, no gaps or discontinuities).

c When visible, the percentage of cases in which the LR-SR band demonstrated superotemporal bowing.

${ }^{\mathrm{d}}$ The percentage of cases in which the lateral levator aponeurosis was visible, distinct from the LR-SR band.

Table 2: Imaging metrics per patient age quartile ${ }^{\mathrm{a}}$

\begin{tabular}{lccccc}
\hline & Quartile 1 & Quartile 2 & Quartile 3 & Quartile 4 & P Value \\
\hline LR-SR visible on TIWT $^{\mathrm{b}}$ & $96 \%$ & $99 \%$ & $95 \%$ & $90 \%$ & .03 \\
LR-SR continuous $^{\mathrm{c}}$ & $95 \%$ & $96 \%$ & $95 \%$ & $91 \%$ & .40 \\
LR-SR bowing $^{\mathrm{d}}$ & $19 \%$ & $17 \%$ & $28 \%$ & $34 \%$ & .03 \\
LLA visible $^{\mathrm{e}}$ & $73 \%$ & $77 \%$ & $89 \%$ & $87 \%$ & .01 \\
\hline
\end{tabular}

Note:-LLA indicates lateral levator aponeurosis.

${ }^{a}$ Quartile 1: 9-39 years of age; quartile 2: 40-49 years of age; quartile 3: 50-61 years of age; quartile 4: 62-81 years of age.

b The percentage of cases in which the LR-SR band was visible on TIWI.

c The percentage of cases in which the LR-SR band was visible as a continuous structure extending from the superior muscle complex to the lateral rectus muscle (ie, no gaps or discontinuities).

${ }^{d}$ The percentage of cases in which the LR-SR band demonstrated superotemporal bowing.

e The percentage of cases in which the lateral levator aponeurosis was visible, distinct from the LR-SR band. results indicate that $\mathrm{CT}$ does, in fact, display the band in a substantial majority of orbits.

Both LR-SR band discontinuity (or "rupture") and superotemporal displacement have been reported as features of band degeneration in patients with strabismus. ${ }^{10,11}$ In our cohort of patients without strabismus, we report the appearance of a noncontinuous LR-SR band in $5 \%$ of cases and superotemporal bowing of the LR-SR band in $24 \%$ of cases. These results indicate that band discontinuity may represent a more specific sign of pathologic band degeneration than superotemporal bowing. LR-SR band "thinning" has also been described as a feature of band degeneration. ${ }^{10,11}$ We found no reliable criteria with which to distinguish "thick" and "thin" LR-SR bands, so we did not assess LR-SR bands for this feature.

Our results demonstrate a negative age effect with respect to LR-SR band visibil-

metrics per age quartile. There was no sex correlation with any of the imaging metrics.

\section{DISCUSSION}

We describe the normal appearance of the LR-SR band on clinical imaging examinations in a large cohort of patients without strabismus, both on 3T MR imaging and CT. Our results indicate that the LR-SR band is a readily visible structure on coronal T1weighted images obtained without fat suppression. Visibility of the band is much less reliable with coronal STIR sequences and coronal CT. When visible on MR imaging, the band appears as a hypointense, arc-like structure extending from the superior margin of the lateral rectus muscle to the lateral margin of the superior rectus/levator palpebrae muscle complex. On CT, the band appears as a soft-tissue attenuation structure conforming to its shape on the corresponding MR imaging. Of note, it has previously been stated that the LR-SR band is not visible on $\mathrm{CT}^{11}$; our ity and a positive age effect with respect to superotemporal bowing of the LR-SR band. These findings may represent normal agerelated connective tissue degeneration and laxity of the LR-SR band. Supportive evidence for this phenomenon comes from histologic investigations by Rutar and Demer, ${ }^{11}$ demonstrating progressive attenuation and superotemporal displacement of the LR-SR band in specimens of progressively older individuals.

The lateral levator aponeurosis is a slip of connective tissue extending from the lateral aspect of the levator palpebrae superioris, through the lacrimal gland, to the orbital wall near the lateral canthus. ${ }^{4}$ This structure is normally located superior and temporal to the LR-SR band. The study by Rutar and Demer ${ }^{11}$ reported the finding that the LR-SR band was indistinguishable from the overlying lateral levator aponeurosis in the specimen of the oldest patient examined histologically but it was distinct from the lateral levator aponeurosis in the other specimens. Thus, we expected the 
lateral levator aponeurosis to be more difficult to visualize in older patients than in younger patients. Our findings showed the opposite result: The lateral levator aponeurosis is easier to see in older individuals. We hypothesize that this is due to increased fat content within the lacrimal gland. On non-fat-suppressed T1weighted images, this provides higher contrast between the hypointense aponeurosis and the surrounding hyperintense lacrimal gland.

Our reported $\kappa$ values are mixed, with some imaging metrics showing low levels of interreader agreement. This finding is not unexpected in the evaluation of a structure not previously characterized in the radiology literature. Moreover, we evaluated a group of nominally healthy (ie, without strabismus) patients. Therefore, abnormalities of the LR-SR band (nonvisibility, bowing, discontinuity) are de-emphasized by design. When present in our patient cohort, these abnormalities are expected to be more subtle and infrequent than those seen in patients with strabismus, also predicting that $\kappa$ values might be negatively affected. However, despite the relatively low $\kappa$ values, the readers independently showed agreement in identifying a continuous LR-SR band in most orbits on T1WI.

In the course of their consensus review, the readers identified several factors that potentially led to the discrepancies

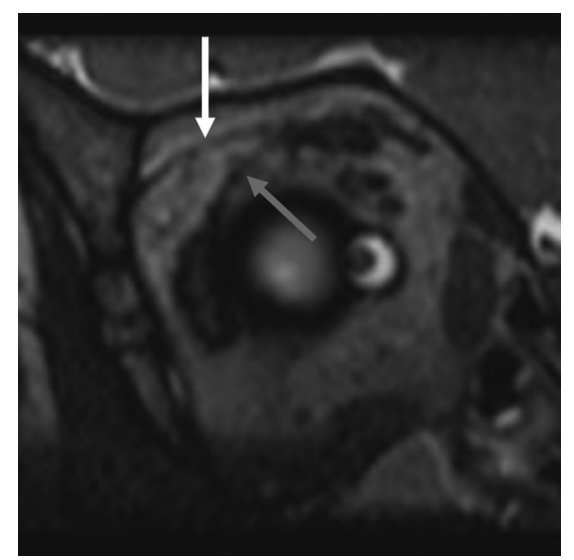

FIG 6. Coronal image of the right orbit from a CISS sequence in a 5-year-old boy displays the LR-SR band (gray arrow) and the lateral levator aponeurosis (white arrow).
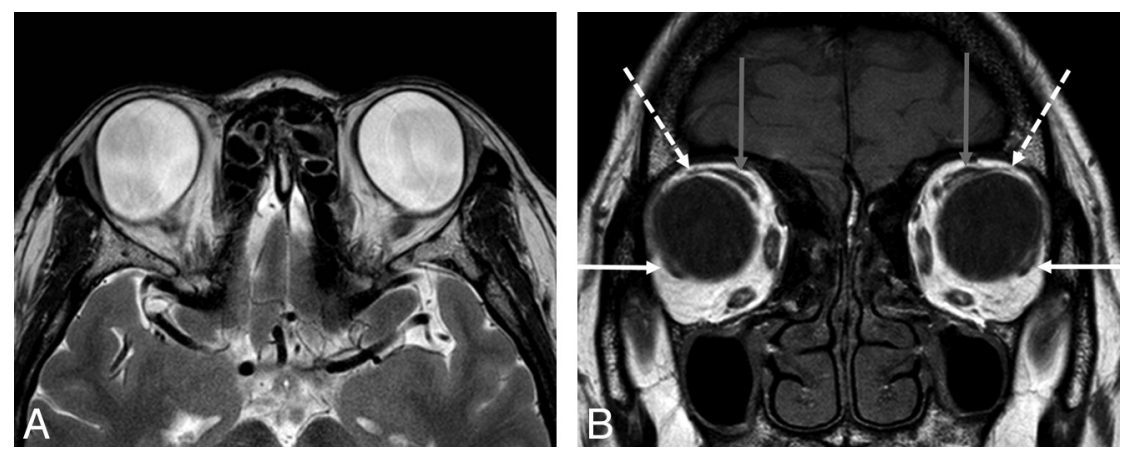

FIG 7. A 65-year-old woman with heavy eye syndrome who presented with high myopia and extreme esotropia (inward eye deviation), resulting in poor peripheral vision. Axial T2WI $(A)$ shows large bilateral staphylomas and esotropia. Coronal TIWI (B) shows thinned/incomplete LR-SR bands (white dashed arrows) stretched over the enlarged globes and nasal displacement of the superior muscle complexes (gray arrows) and inferior displacement of the lateral rectus muscles (white solid arrows). (and which, in general, may complicate the confident identification of the LR-SR band). These factors included the following: the close apposition of the LR-SR band to the globe surface, inhomogeneous orbital fat, patient motion, and very thin bands.

There are limitations to our study. No histologic correlate was provided for the structures determined to represent the LR-SR band and the lateral levator aponeurosis. Thus, in cases in which the LR-SR band was not visible, it is unclear whether the band was not present or whether the lack of visibility represented a limitation in the sensitivity of our imaging technique. It is also possible that orbital connective tissues are better imaged with MR imaging sequences other than those evaluated in this study. For instance, in our limited experience, we have found that steady-state highresolution T2-weighted sequences (eg, CISS or FIESTA) can provide excellent delineation of the LR-SR band and the lateral levator aponeurosis, with thin-section reconstructions and no interection gap (Fig 6). Finally, CT scans were available in only a minority of our patients. Nonetheless, it seems clear from our experience that $\mathrm{CT}$ is far less reliable in imaging orbital connective tissues than MR imaging, mainly due to worse contrast resolution.

The role of imaging the LR-SR band in clinical practice remains unclear. While heavy eye syndrome and sagging eye syndrome are diagnosed mainly on clinical grounds, early reports indicate that orbital MR imaging may add diagnostic specificity in the work-up of these disorders. In particular, it can readily clinch the diagnosis of heavy eye syndrome (Fig 7). Imaging is also useful in excluding other causes of strabismus (mass lesions, infarcts, and so forth). It is conceivable that imaging findings might help stratify patients with strabismus in whom the outcomes of surgical therapies differ. Further studies will be required to answer such questions and confirm the clinical use of imaging in the management of heavy eye and sagging eye syndromes.

Other questions of potential clinical import relate to the significance of the LR-SR band beyond its physiologic role as a suspensory ligament. For instance, it is possible that the LR-SR band constrains the spread of orbital pathology in a manner analogous to the orbital septum. ${ }^{14,15}$ Anecdotally, we have observed several cases of extraconal orbital tumors that seem to displace, but not clearly transgress, the LR-SR band (Fig 8). Perhaps some pathologies more frequently displace and/or transgress the LR-SR band than others. If so, these distinctions might improve diagnostic specificity when evaluating orbital lesions on imaging studies and affect surgical approaches to orbital lesions.

\section{CONCLUSIONS}

The LR-SR band is readily visible in clinical orbital imaging studies of patients without strabismus. The LR-SR band is seen in 95\% of orbits on coronal T1weighted images obtained without fat 


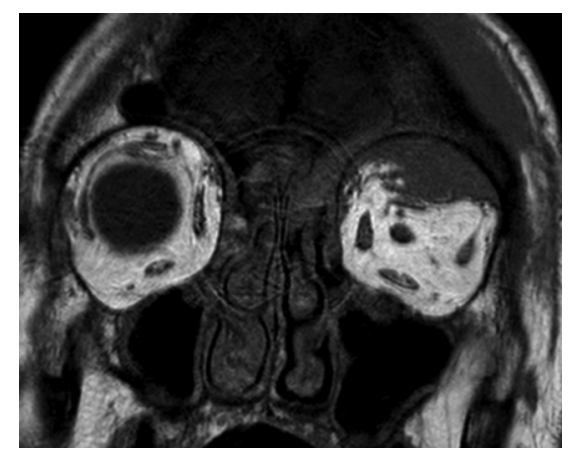

FIG 8. A 74-year-old man with B-cell lymphoma. Coronal precontrast T1-weighted image demonstrates lymphoma involving the superotemporal left orbit. Tumor appears to cause inferior bowing/displacement of the LR-SR band but does not definitely transgress it. Note the normal LR-SR band in the right orbit.

suppression. It nearly always appears as an uninterrupted, arc-like structure extending from the lateral rectus to the superior rectus/ levator palpebrae muscle complex. There is an age effect with respect to visibility and superotemporal bowing of the LR-SR band, possibly representing normal age-related connective tissue degeneration of the band. The lateral levator aponeurosis is more easily visible with increasing age, possibly due to increased fat content within the lacrimal glands of older individuals.

Disclosures: Melanie A. Kazlas_UNRELATED: Employment: I am a pediatric ophthalmologist employed by Boston Children's Ophthalmology Foundation, Grants/ Grants Pending: Children's Hospital Boston Ophthalmology Foundation In-House Grant of $\$ 25,000$ to study effect of light deprivation in amblyopia, Payment for Lectures (including service on Speakers Bureaus): invited lecturer, Ophthalmic Anesthesia Society (September 11, 2013). Gul Moonis_UNRELATED: Other: Grand Rounds Beth Israel, New York (honorarium).

\section{REFERENCES}

1. Koornneef L. New insights in the human orbital connective tissue: result of a new anatomical approach. Arch Ophthalmol 1977;95: $1269-73$

2. Koornneef L. Details of the orbital connective tissue system in the adult. Acta Morphol Neerl Scand 1977;15:1-34

3. Demer JL. Pivotal role of orbital connective tissues in binocular alignment and strabismus: the Friedenwald lecture. Invest Ophthalmol Vis Sci 2004;45:729-38

4. Kono R, Poukens V, Demer JL. Quantitative analysis of the structure of the human extraocular muscle pulley system. Invest Ophthalmol Vis Sci 2002;43:2923-32

5. Miller JM. Understanding and misunderstanding extraocular muscle pulleys. J Vis 2007;7:11-15

6. Miller JM, Demer JL, Poukens V, et al. Extraocular connective tissue architecture. J Vis 2003;3:240-51

7. Miller JM. Functional anatomy of normal human rectus muscles. Vision Res 1989;29:223-40

8. Clark RA, Miller JM, Demer JL. Three-dimensional location of human rectus pulleys by path inflections in secondary gaze positions. Invest Ophthalmol Vis Sci 2000;41:3787-97

9. Demer JL. The orbital pulley system: a revolution in concepts of orbital anatomy. Ann N Y Acad Sci 2002;956:17-32

10. Chaudhuri Z, Demer JL. Sagging eye syndrome: connective tissue involution as a cause of horizontal and vertical strabismus in older patients. JAMA Ophthalmol 2013;131:619-25

11. Rutar T, Demer JL. "Heavy eye" syndrome in the absence of high myopia: a connective tissue degeneration in elderly strabismic patients. J AAPOS 2009;13:36-44

12. Kowal L, Troski M, Gilford E. MRI in the heavy eye phenomenon. Aust N Z J Ophthalmol 1994;22:125-26

13. $\mathrm{R}$ Core Team. R: A language and environment for statistical computing. $R$ Foundation for Statistical Computing 2013. www.Rproject.org

14. Botting AM, McIntosh D, Mahadevan M. Paediatric pre- and postseptal peri-orbital infections are different diseases: a retrospective review of 262 cases. Int J Pediatr Otorhinolaryngol 2008;72:377-83

15. Wald ER. Periorbital and orbital infections. Pediatr Rev 2004; 25:312-20 